Article

\title{
Synthesis and Disinfection Effect of the Pyridine-4-aldoxime Based Salts
}

\author{
Jan Marek ${ }^{1,2}$, David Malinak ${ }^{3,4}$, Rafael Dolezal ${ }^{3}$, Ondrej Soukup ${ }^{3,5}$, Marketa Pasdiorova ${ }^{2,3}$, \\ Martin Dolezal ${ }^{1}$ and Kamil Kuca ${ }^{3,5, *}$
}

1 Department of Medicinal Chemistry and Drug Analysis, Faculty of Pharmacy, Charles University in Prague, Heyrovskeho 1203, Hradec Kralove 500 05, Czech Republic;

E-Mails: marekjanmgr@seznam.cz (J.M.); martin.dolezal@faf.cuni.cz (M.D.)

2 Department of Epidemiology, Faculty of Military Health Sciences, University of Defence, Trebesska 1575, Hradec Kralove 500 01, Czech Republic; E-Mail: MarketaPasdiorova@seznam.cz

3 Biomedical Research Centre, University Hospital Hradec Kralove, Sokolska 581, Hradec Kralove 500 05, Czech Republic; E-Mails: david.malinak@gmail.com (D.M.); rafael.dolezal@fnhk.cz (R.D.); soukup.ondrej07@gmail.com (O.S.)

4 Department of Cybernetics and Biomedical Engineering, Faculty of Electrical Engineering and Computer Science, VSB-Technical University of Ostrava, 17. Listopadu 15, Ostrava-Poruba 708 33, Czech Republic

5 Department of Toxicology and Military Pharmacy, Faculty of Military Health Sciences, University of Defence, Trebesska 1575, Hradec Kralove 500 01, Czech Republic

* Author to whom correspondence should be addressed; E-Mail: kamil.kuca@fnhk.cz; Tel.: +420-495-832-923.

Academic Editor: Peter J. Rutledge

Received: 4 January 2015 / Accepted: 12 February 2015 / Published: 24 February 2015

\begin{abstract}
A set of new quaternary ammonium compounds based on pyridine-4-aldoxime was synthesized, characterized with analytical data (NMR, EA, HPLC, MS) and tested for in vitro antimicrobial activity (antibacterial, antifungal) and cytotoxicity. Quaternary pyridinium-4-aldoxime salts with length of alkyl side chain from C8 to C20 and belonging to the group of cationic surfactants were investigated in this work. An HPLC experimental protocol for characterization of mixtures of all homologues has been found. Antimicrobial evaluation found that yeast-type fungi were most sensitive towards $\mathrm{C}_{14}$ and $\mathrm{C}_{16}$ analogues, whereas the $\mathrm{C}_{16}$ analogue was completely ineffective against filamentous fungi. Antibacterial assessment showed versatility of $\mathrm{C}_{14}$ and relatively high efficacy of $\mathrm{C}_{16}$
\end{abstract}


against $\mathrm{G}^{+}$strains and $\mathrm{C}_{14}$ against $\mathrm{G}^{-}$strains. Notably, none of the studied compounds exceeded the efficacy and versatility of the benzalkonium $\mathrm{C}_{12}$ analogue, and benzalkonium analogues also exhibited lower cytotoxicity in the cell viability assay.

Keywords: pyridinium-4-aldoxime salts; synthesis; analysis; antimicrobial activity; surfactants; cytotoxicity

\section{Introduction}

The 20th century can appropriately be called the age of organic chemistry. It brought many new organic structures, both natural and synthetic. Here the discussed surfactants are undoubtedly a huge group of organic compounds for which a technical use has been found. These compounds have certainly undergone considerable development since their discovery in 1930, and countless applications have been reported for these important molecules (e.g., detergents, disinfectants, decontaminants, etc.) [1-3].

Cationic surfactants consist of a hydrophilic part, such as a quaternary nitrogen moiety, able to interact with polar chemical milieu, and a hydrophobic part (e.g., a long alkyl chain), which can, on the other hand, penetrate into non-polar molecular agglomerates.

Many types of cationic surfactant based on quaternary nitrogen have been described [4-6]. One important property described for quaternary ammonium salts is inhibition of the growth of bacteria and fungi. The most commonly used are derivatives of pyridinium, cetrimonium, benzalkonium and benzoxonium salts [7-10]. Some pyridinium salts are already in use as disinfectants in many preparations (as a component of eye drops, solutions, disinfection foams, etc.). Currently, there have been many articles describing cetylpyridinium salt as a protective agent against food-borne disease [6]. Recently, polymeric quaternary ammonium salts have been used in the development of bactericidal surfaces [11,12]. Another antimicrobial application of cetylpyridinium is its addition to chewing gums or dental formulations as an antiplaque agent $[13,14]$. In order to intensify the development of cetylpyridinium analogues, the $2 \mathrm{D}$ and $3 \mathrm{D}$ QSAR method was established [6]. It was found that numerous properties such as substituents on the pyridine moiety, alkyl side-chain length, hydrophobicity, pKa and absorbability on to the cell are critically important for antimicrobial activity $[15,16]$.

Quaternary ammonium salts (QAS) are also frequently used in prevention of nosocomial infections that are predominantly acquired within healthcare facilities, and conditioned mainly by the impaired immune status of patients, absence of a strict glove-change regime, and the use of improperly or inadequately sterilized medical instruments [17]. An important property of such compounds is their ability to form micelles (Figure 1). These formations are created mainly in aqueous solution, when the critical micelle concentration is exceeded. Many cationic surfactants can also be used as micellar catalysts [18], able to accelerate chemical decomposition [19,20], and such compounds can find use as decontaminants for military purposes. Thus these properties are important in decontamination procedures for measuring the kinetics of cleavage of some model compounds such as organophosphates and others [21-26]. Many other properties of cationic surfactants were discovered. Enthalpies of dilution, density effects of counterions in micellar solution and osmotic coefficients have 
been measured as a function of concentration, and the results expressed in terms of partial molar quantities [27-29].

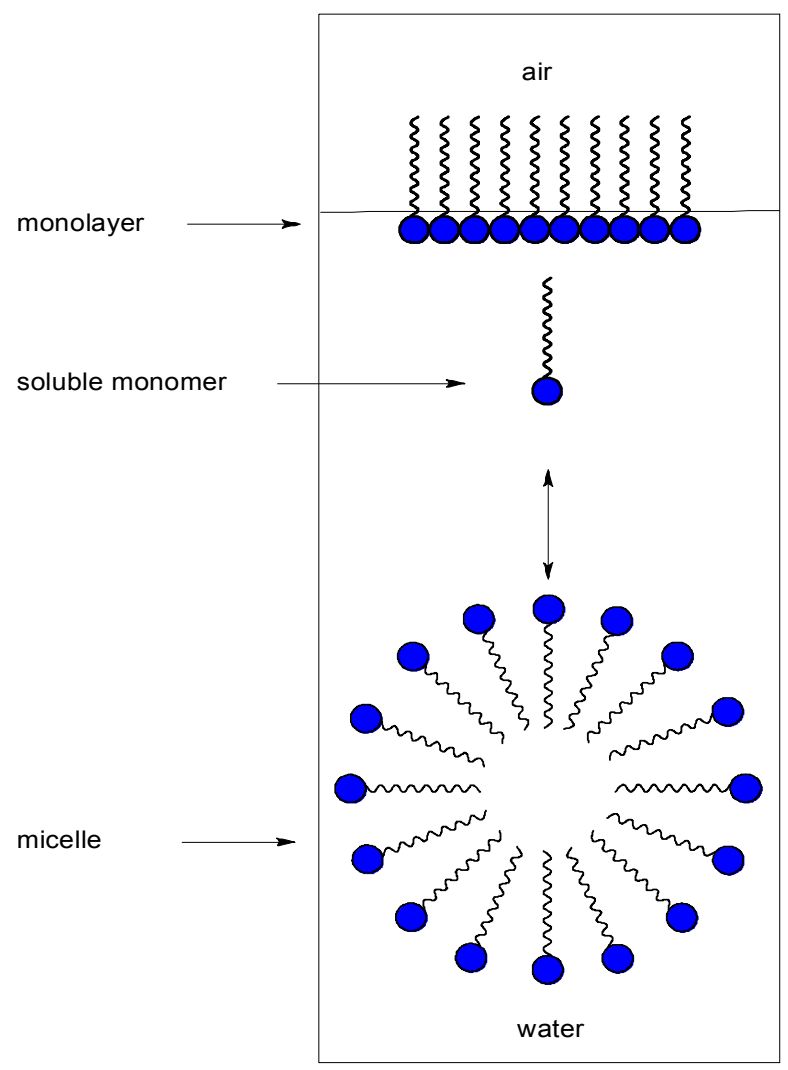

Figure 1. Micelles.

Our group has been devoted to the synthesis and evaluation of derivatives of heterocyclic nitrogen compounds [30-33] and tensides [34-36]. Preparation of the various pyridinium-4-aldoxime salts has already been described before by our group, where the compounds were evaluated as micellar catalysts $[24,25]$. However, there has been no description of the synthesis of the whole series of such salts differing in the alkyl chain $\left(\mathrm{C}_{8}\right.$ to $\left.\mathrm{C}_{20}\right)$, and their antimicrobial properties have not been measured. The primary aim of this work was to investigate this group of QAS as potential affordable disinfectants. For this reason a universal method was developed for preparation of monoquaternary pyridinium-4-aldoxime salts with alkyl-chain substituents, and the prepared compounds were characterized using analytical data (NMR, EA, HPLC, MS) and tested for in vitro antimicrobial activity and cytotoxicity.

\section{Results and Discussion}

\subsection{Synthesis and Analysis}

The results achieved within our study are shown in Table 1. This includes yields, melting points, retention times obtained on HPLC and calculated $\log P(\mathrm{C} \log P)$. It is evident that the preparation of pyridinium-4-aldoxime salts with side chain $\mathrm{C}_{8}-\mathrm{C}_{20}$ is a quite elementary one step reaction. On the other hand, repeated crystallization was necessary in order to achieve the required purity. Two different conditions for synthesis were used. The reactions were realized in two different solutions, ethanol $(\mathrm{EtOH})$ or acetonitrile $\left(\mathrm{CH}_{3} \mathrm{CN}\right)$ (Scheme 1), and the prepared compounds 9-15 were 
recrystallized from ethyl acetate or acetone. There was no significant variation in the yields from both solvents. Yields were highest for compounds 11-13. A comparison can be made of the influence of the non-polar part of molecule on the yields during the quaternization of $N, N$-dimethyl- $N$-benzylamine, pyridine or derivatives of quinoline $[31,33,37,38]$. As expected, the value of $\operatorname{Clog} P$ increases with the length of the non-polar chain.

Table 1. Yields, melting points and retention times of prepared pyridinium-4-aldoxime salts.

\begin{tabular}{ccccccc}
\hline Comp. & $\mathbf{R}$ & Yields (\%) EtOH & Yields (\%) $\mathbf{C H}_{\mathbf{3}} \mathbf{C N}$ & m.p. $\left({ }^{\circ} \mathbf{C}\right)$ & HPLC Rt (min) & ClogP \\
\hline $\mathbf{9}$ & $\mathrm{C}_{8}$ & 15 & $\mathrm{X}$ & $92-93$ & 4.15 & 0.04 \\
$\mathbf{1 0}$ & $\mathrm{C}_{10}$ & 27 & 41 & $130-132$ & 4.67 & 1.10 \\
$\mathbf{1 1}$ & $\mathrm{C}_{12}$ & 70 & 55 & $136-137$ & 5.22 & 2.15 \\
$\mathbf{1 2}$ & $\mathrm{C}_{14}$ & 72 & 75 & $144-146$ & 5.82 & 3.21 \\
$\mathbf{1 3}$ & $\mathrm{C}_{16}$ & 75 & 87 & $144-145$ & 6.49 & 4.27 \\
$\mathbf{1 4}$ & $\mathrm{C}_{18}$ & 47 & 76 & $128-130$ & 7.23 & 5.33 \\
$\mathbf{1 5}$ & $\mathrm{C}_{20}$ & 45 & $\mathrm{X}$ & $126-128$ & 8.06 & 6.39 \\
\hline
\end{tabular}

Note: $\mathrm{X}-$ Not prepared in $\mathrm{CH}_{3} \mathrm{CN}$.
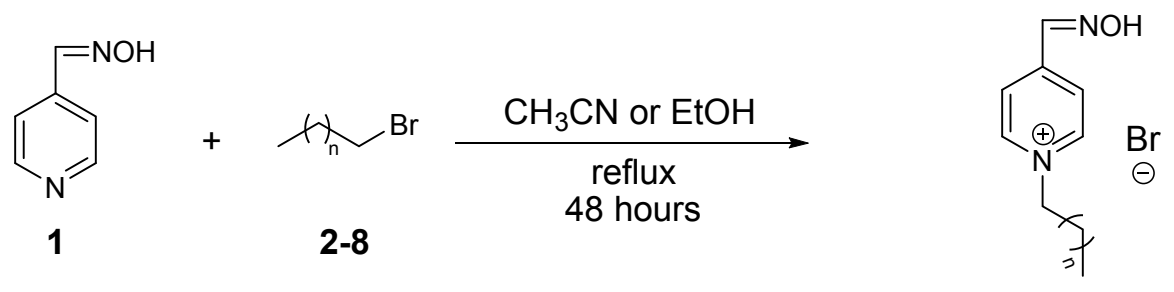

$\mathrm{n}=6,8,10,12,14,16,18$

9-15

Scheme 1. Preparation of pyridinium-4-aldoxime salts.

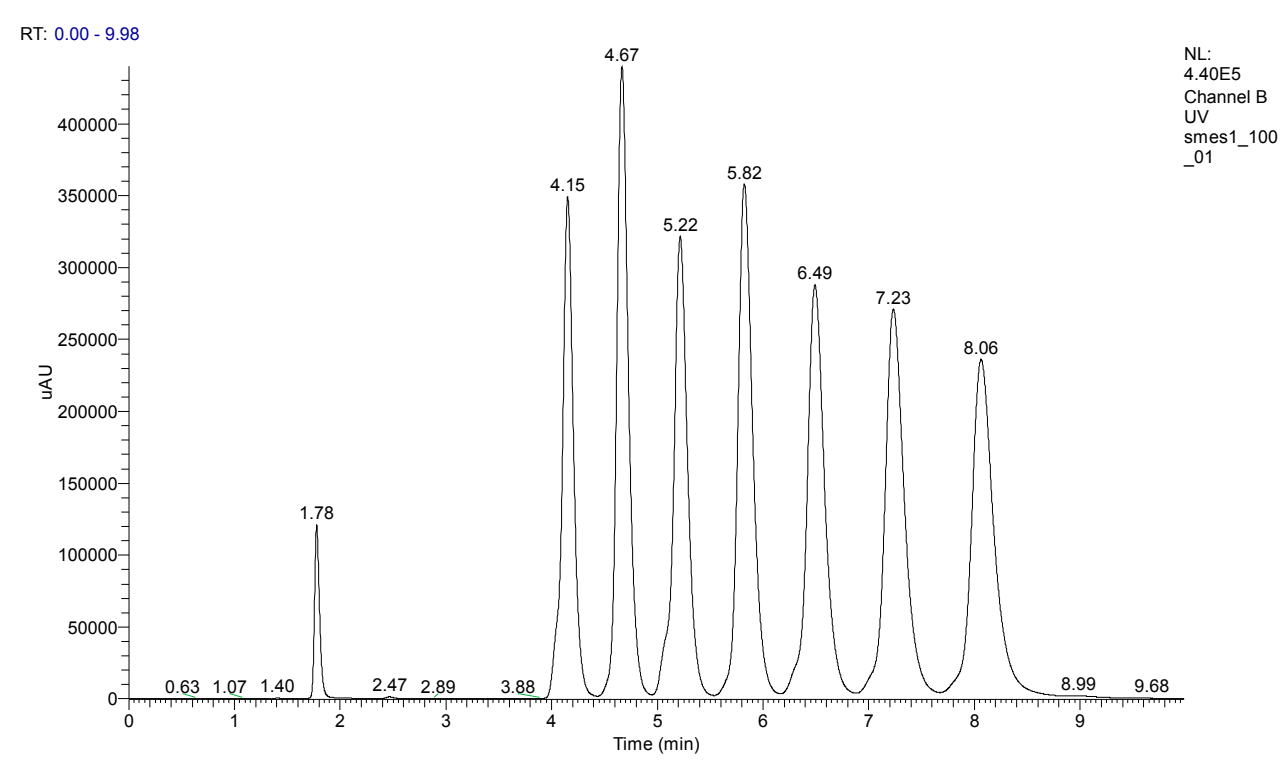

Figure 2. HPLC chromatogram of pyridinium-4-aldoxime salts mixture.

Additionally, HPLC analysis was carried out for individual compounds. A newly developed HPLC method was able to distinguish all prepared quaternary pyridinium-4-aldoxime salts (Figure 2). The 
shortest retention time was found for the $\mathrm{C}_{8}$ pyridinium-4-aldoxime salt. This novel HPLC method could be easily used for characterization of mixtures of all homologues.

\subsection{Antimicrobial Activity}

A set of eight fungus strains was used to evaluate antifungal activity. The minimum inhibitory concentrations (MICs) and minimum fungicidal (MFCs) concentrations for four yeasts and four filamentous fungi are summarized in Table 2. The whole set of pyridinium-4-aldoxime salts was tested for biological activity. The results for compounds $\mathbf{9 - 1 5}$ as well as for the reference benzalkonium $\mathrm{B}_{12}$, $\mathrm{B}_{14}$ and $\mathrm{B}_{16}$ compounds are shown in Table 2 .

A broad spectrum of activity is evident for compounds 11-14. The CK strain is sensitive for the whole set of pyridinium-4-aldoxime salts except for compound $\mathbf{1 5}$ ( $\mathrm{C}_{20}$ alkyl chain). The yeast-type strains (CA, CT, CK, CG) are most sensitive to compounds 12-14. The effectiveness is comparable with the benzalkonium salts. However, there is no clear correlation with the alkyl chain length.

The disruptive effect of QAS on the microorganism is probably based on the adsorption of this amphiphile molecule on the outer cellular membrane. The positively charged "heads" of the cationic molecules interact with the negatively charged cell membrane, disrupting it thanks to electrostatic and hydrophobic interactions. These interactions effectively out-compete the divalent cations, which normally stabilize surface structures by linking adjacent negatively-charged components [39]. Once close contact is accomplished by the hydrophilic region, the hydrophobic region proceeds to penetrate the hydrophobic bilayer to cause cell leakage and lysis [40]. This cascade leads to the release of $\mathrm{K}^{+}$and cytoplasmic components, and finally to death of the cell. The antibacterial activity thus depends on the hydrophilic-hydrophobic balance of the cationic surfactants. Increase in the alkyl chain length (from $\mathrm{C}_{12}$ to $\mathrm{C}_{16}$ ) increases the hydrophobic character, which may be too high to facilitate transport through the bacterial cell membrane [41].

The filamentous fungi strains have significant sensitivity only to compounds $\mathbf{1 2}$ and $\mathbf{1 3}$ (alkyl length $\mathrm{C}_{14}$ and $\mathrm{C}_{16}$ ). The values of MICs and MFCs are higher compared to benzalkonium reference salts. The other compounds have no or only minor efficacy against filamentous fungi.

The in vitro antibacterial activity of compounds 9-15 was also assayed for the eight strains of bacteria. Gram positive $\left(\mathrm{G}^{+}\right)$and gram-negative $\left(\mathrm{G}^{-}\right)$bacteria groups are represented to cover the entire spectrum. Listed in Table 3 are the obtained minimum inhibitory concentrations (MICs) after $24 \mathrm{~h}$ and $48 \mathrm{~h}$ of incubation, and the minimum bactericidal concentrations (MBCs) after $48 \mathrm{~h}$ of incubation. Again, commercially used benzalkonium salts with alkyl side-chain length $\mathrm{C}_{12}, \mathrm{C}_{14}$ and $\mathrm{C}_{16}$ are included for comparison of antibacterial activity ( $\left.\mathrm{B}_{12}, \mathrm{~B}_{14}, \mathrm{~B}_{16}\right)$.

In general, the relationship of structure and antibacterial activity shows the most efficient compounds to be 11-13 (alkyl chain $\mathrm{C}_{12}, \mathrm{C}_{14}, \mathrm{C}_{16}$ ), highlighting the versatility of $\mathbf{1 2}$ and the high efficacy of 13 against $\mathrm{G}+$ strains. The other compounds with a shorter or longer lipophilic chain did not show any distinctive effect. Only the relatively high potency of 11, 14 and 15 against SE can be noted. It was found that the Gram-positive bacteria (first four strains) are most sensitive to compounds $\mathbf{1 2}$ and $\mathbf{1 3}$ (alkyl chain $\mathrm{C}_{14}$ and $\mathrm{C}_{16}$ ). In comparison with the benzalkonium salts, the effectiveness of compound $\mathbf{1 3}$ seems to be better (MBC) against SA. The sensitivity of the other strains of $\mathrm{G}+$ bacteria is comparable or lower. 
Table 2. Minimum inhibitory/fungicidal concentrations of the prepared compounds (9-15) and selected derivatives of $N$-benzalkonium $\left(\mathrm{B}_{12}-\mathrm{B}_{16}\right)$. The in vitro activities were determined on a panel of eight fungal strains.

\begin{tabular}{|c|c|c|c|c|c|c|c|c|c|c|}
\hline \multirow{3}{*}{ Microorganisms } & \multicolumn{10}{|c|}{ MIC $(\mu \mathrm{mol} / \mathrm{L}) ; 24 \mathrm{~h} / 48 \mathrm{~h}$ Incubation } \\
\hline & \multicolumn{10}{|c|}{ MFC $(\mu \mathrm{mol} / \mathrm{L}) ; 48 \mathrm{~h}$ Incubation } \\
\hline & 9 & 10 & 11 & 12 & 13 & 14 & 15 & $\mathrm{~B}_{12}{ }^{\mathrm{a}}$ & $\mathrm{B}_{14}{ }^{\mathrm{a}}$ & $\mathrm{B}_{16}{ }^{\mathrm{a}}$ \\
\hline Candida albicans ATCC & $>500 />500$ & $125 / 250$ & $15.62 / 15.62$ & $1.95 / 1.95$ & $3.9 / 3.9$ & $7.81 / 7.81$ & $>500 />500$ & $0.49 / 0.49$ & $7.81 / 7.81$ & $3.91 / 7.81$ \\
\hline $44859(\mathrm{CA})$ & $>500$ & 250 & 15.62 & 1.95 & 3.9 & 15.62 & $>500$ & 0.98 & 125 & 7.81 \\
\hline \multirow{2}{*}{ Candida tropicalis $156(\mathrm{CT})$} & $125 / 125$ & $31.25 / 31.25$ & $7.81 / 7.81$ & $3.9 / 3.9$ & $3.9 / 3.9$ & $7.81 / 7.81$ & $>500 />500$ & $0.49 / 0.49$ & $3.91 / 3.91$ & $3.91 / 7.81$ \\
\hline & 250 & 125 & 31.25 & 15.62 & 3.9 & 15.62 & $>500$ & 0.98 & 125 & 7.81 \\
\hline \multirow{2}{*}{ Candida krusei E28 (CK) } & $31.25 / 62.5$ & $3.9 / 3.9$ & $0.98 / 1.95$ & $1.95 / 1.95$ & $3.9 / 3.9$ & $3.9 / 3.9$ & $>500 />500$ & $0.49 / 0.49$ & $3.91 / 3.91$ & $1.95 / 1.95$ \\
\hline & 125 & 15.62 & 3.9 & 15.62 & 3.9 & 15.62 & $>500$ & 0.49 & 125 & 1.95 \\
\hline \multirow{2}{*}{ Candida glabrata 20/I (CG) } & $125 / 125$ & $31.25 / 31.25$ & $3.9 / 3.9$ & $1.95 / 3.9$ & $3.9 / 3.9$ & $3.9 / 3.9$ & $>500 />500$ & $0.49 / 0.49$ & $7.81 / 7.81$ & $1.95 / 3.91$ \\
\hline & 250 & 62.5 & 3.9 & 31.25 & 3.9 & 7.81 & $>500$ & 0.49 & 125 & 62.5 \\
\hline Trichosporon asahii & $>500>500$ & $500 />500$ & $125 / 125$ & $15.62 / 15.62$ & $7.81 / 7.81$ & $7.81 / 7.81$ & $>500 />500$ & $0.49 / 1.95$ & $31.25 / 31.25$ & $7.81 / 7.81$ \\
\hline $1188(\mathrm{TA})$ & $>500$ & $>500$ & 500 & 31.25 & 7.81 & 7.81 & $>500$ & 1.95 & 125 & 62.5 \\
\hline Aspergillus fumigatus & $>500 />500$ & $>500 />500$ & $125 / 250$ & $62.5 / 62.5$ & $15.62 / 15.62$ & $>500 />500$ & $>500 />500$ & $0.98 / 3.91$ & $7.81 / 15.62$ & $7.81 / 7.81$ \\
\hline $231(\mathrm{AF})$ & $>500$ & $>500$ & $>500$ & 250 & 62.5 & $>500$ & $>500$ & 3.91 & 125 & 62.5 \\
\hline Absidia corymbifera & $>500 />500$ & $>500 />500$ & $500 / 500$ & $62.5 / 62.5$ & $15.62 / 31.25$ & $>500 />500$ & $>500 />500$ & $7.81 / 7.81$ & $31.25 / 31.25$ & $7.81 / 7.81$ \\
\hline $272(\mathrm{AC})$ & $>500$ & $>500$ & 500 & 500 & 62.5 & $>500$ & $>500$ & 7.81 & 125 & 62.5 \\
\hline Trichophyton & $>500>500$ & $>500 />500$ & $62.5 / 62.5$ & $15.62 / 62.5$ & $15.62 / 15.62$ & $>500 />500$ & $>500 />500$ & $0.98 / 0.98$ & $15.62 / 15.62$ & $7.81 / 7.81$ \\
\hline mentagrophytes 445 (TM) & $>500$ & $>500$ & 125 & 125 & 62.5 & $>500$ & $>500$ & 1.95 & 15.62 & 62.5 \\
\hline
\end{tabular}

Notes: $\quad{ }^{a} \quad \mathrm{~B}_{12}, \quad \mathrm{~B}_{14}, \quad \mathrm{~B}_{16}$ mean $N$-benzyl- $N, N$-dimethyl- $N$-dodecylammonium bromide, $N$-benzyl- $N, N$-dimethyl- $N$-tetradecylammonium bromide, $N$-benzyl- $N$, $N$-dimethyl- $N$-hexadecylammonium bromide, respectively. The preparation [33] and antimicrobial efficacy of $\mathrm{B}_{12-16}$ has been published elsewhere. The antifungal activities against Trichophyton mentagrophytes 445 were determined after $72 \mathrm{~h}$ and $120 \mathrm{~h}$ of incubation. 
Table 3. Minimum inhibitory/bactericidal concentrations of the prepared compounds (9-15) and selected derivatives of $N$-benzalkonium $\left(\mathrm{B}_{12}-\mathrm{B}_{16}\right)$. The in vitro activities were determined on a panel of eight bacterial strains.

\begin{tabular}{|c|c|c|c|c|c|c|c|c|c|c|}
\hline \multirow{3}{*}{ Microorganisms } & \multicolumn{10}{|c|}{ MIC $(\mu \mathrm{mol} / \mathrm{L}) ; 24 \mathrm{~h} / 48 \mathrm{~h}$ Incubation } \\
\hline & \multicolumn{10}{|c|}{ MBC $(\mu \mathrm{mol} / \mathrm{L}) ; 48 \mathrm{~h}$ Incubation } \\
\hline & 9 & 10 & 11 & 12 & 13 & 14 & 15 & $\mathrm{~B}_{12}{ }^{\mathrm{a}}$ & $\mathrm{B}_{14}{ }^{\mathrm{a}}$ & $\mathrm{B}_{16}{ }^{\mathrm{a}}$ \\
\hline Staphylococcus aureus & $125 / 125$ & $15.62 / 15.62$ & $15.62 / 15.62$ & $1.95 / 7.81$ & $0.98 / 0.98$ & $7.81 / 7.81$ & $15.62 / 15.62$ & $0.49 / 1.95$ & $0.98 / 0.98$ & $0.98 / 0.98$ \\
\hline CCM 451608 (SA) & 125 & 15.62 & 15.62 & 7.81 & 0.98 & 7.81 & 15.62 & 1.95 & 3.91 & 3.91 \\
\hline Staphylococcus aureus $\mathrm{H}$ & $3.9 / 15.62$ & $31.25 / 31.25$ & $7.81 / 15.62$ & $0.49 / 3.9$ & $0.98 / 7.81$ & $15.62 / 125$ & $62.5 />250$ & $0.49 / 0.49$ & $1.95 / 1.95$ & $1.95 / 1.95$ \\
\hline 599608 (MRSA) & 15.62 & 31.25 & 15.62 & 7.81 & 7.81 & 125 & $>250$ & 0.98 & 3.91 & 3.91 \\
\hline Staphylococcus epidermidis & $62.5 / 125$ & $15.62 / 15.62$ & $0.98 / 1.95$ & $0.98 / 0.98$ & $0.98 / 0.98$ & $1.95 / 1.95$ & $1.95 / 1.95$ & $0.49 / 0.49$ & $0.98 / 0.98$ & $0.49 / 0.49$ \\
\hline H 696608 (SE) & 250 & 15.62 & 1.95 & 0.98 & 0.98 & 1.95 & 1.95 & 0.49 & 0.98 & 3.91 \\
\hline Enterococcus sp. & $500 />500$ & $62.5 / 125$ & $31.25 / 31.25$ & $0.98 / 7.81$ & $7.81 / 7.81$ & $3.9 / 15.62$ & $62.5 / 62.5$ & $0.49 / 0.98$ & $1.95 / 1.95$ & $1.95 / 1.95$ \\
\hline $\mathrm{J} 1436508$ (ES) & $>500$ & 500 & 62.5 & 7.81 & 7.81 & 15.62 & 62.5 & 0.98 & 7.81 & 3.91 \\
\hline Escherichia coli & $>500 />500$ & $500 / 500$ & $62.5 / 62.5$ & $15.62 / 15.62$ & $15.62 / 15.62$ & $>500 />500$ & $>250 />250$ & $0.49 / 1.95$ & $7.81 / 7.81$ & $7.81 / 7.81$ \\
\hline CCM 4517 (EC) & $>500$ & 500 & 62.5 & 15.62 & 15.62 & $>500$ & $>250$ & 1.95 & 7.81 & 7.81 \\
\hline Klebsiella pneumoniae $\mathrm{D}$ & $>500 />500$ & $500 / 500$ & $62.5 / 62.5$ & $15.62 / 15.62$ & $15.62 / 15.62$ & $>500 />500$ & $>250 />250$ & $0.49 / 0.49$ & $7.81 / 7.81$ & $7.81 / 7.81$ \\
\hline $1175008(\mathrm{KP})$ & $>500$ & 500 & 62.5 & 15.62 & 15.62 & $>500$ & $>250$ & 0.49 & 7.81 & 7.81 \\
\hline Klebsiella pneumoniae J & $>500 />500$ & $>500 />500$ & $125 / 125$ & $15.62 / 15.62$ & $31.25 / 31.25$ & $>500 />500$ & $>250 />250$ & $0.98 / 0.98$ & $7.81 / 7.81$ & $7.81 / 7.81$ \\
\hline 1436808 (KP-E)) & $>500$ & $>500$ & 125 & 15.62 & 31.25 & $>500$ & $>250$ & 0.98 & 7.81 & 7.81 \\
\hline Pseudomonas aeruginosa & $>500 />500$ & $500 / 500$ & $125 / 125$ & $15.62 / 15.62$ & $250 / 250$ & $>500 />500$ & $>250 />250$ & $3.91 / 3.91$ & $15.62 / 31.25$ & $15.62 / 31.25$ \\
\hline CCM $1961(\mathrm{PA})^{\mathrm{c}}$ & $>500$ & 500 & 125 & 15.62 & 250 & $>500$ & $>250$ & 7.81 & 62.5 & 125 \\
\hline
\end{tabular}

Notes: a $\quad \mathrm{B}_{12}, \quad \mathrm{~B}_{14}, \quad \mathrm{~B}_{16}$ mean $N$-benzyl- $N, N$-dimethyl- $N$-dodecylammonium bromide, $N$-benzyl- $N, N$-dimethyl- $N$-tetradecylammonium bromide, $N$-benzyl- $N$, $N$-dimethyl- $N$-hexadecylammonium bromide, respectively. The preparation [33] and antimicrobial efficacy of $\mathrm{B}_{12-16}$ has been published elsewhere. The antibacterial activities against Pseudomonas aeruginosa CCM 1961 were determined after $72 \mathrm{~h}$ and $120 \mathrm{~h}$ of incubation. 
The sensitivity of $\mathrm{G}^{-}$bacteria (the last four strains) is much lower compared to benzalkonium salts. The lower potency of compounds 11-13 is evident (higher MIC and MBC). However, an unexpected sensitivity of PA to compound 12 was observed. With few exceptions, compounds 9, 10, 14 and 15 showed no potency against $\mathrm{G}-$ bacteria.

\subsection{Cytotoxicity}

Cell viability assay confirmed an expected trend that increasing length of carbon chain results in higher cytotoxicity, probably due to higher lipophilicity of the drug, which facilitates penetration into the cell. Comparing the cytotoxic potential of individual analogues, none of the new compounds exceeded its benzalkonium analogue $\left(\mathrm{B}_{12}\right)$ when it comes to the safety potential (Table 4).

Table 4. Cytotoxic potential of the prepared compounds (9-15) and selected derivatives of $N$-benzalkonium $\left(\mathrm{B}_{12}-\mathrm{B}_{16}\right)$. The in vitro activities are expressed as $\mathrm{IC}_{50}(\mu \mathrm{mol} / \mathrm{L}) \pm \mathrm{SEM}$ $(n=3)$.

\begin{tabular}{|c|c|c|c|c|c|c|c|c|c|c|}
\hline \multirow{2}{*}{ Cell Line } & \multicolumn{10}{|c|}{$\mathrm{IC}_{50}(\mu \mathrm{mol} / \mathrm{L}) ; 24 \mathrm{~h}$ Incubation \pm SEM } \\
\hline & 9 & 10 & 11 & 12 & 13 & 14 & 15 & $\mathrm{~B}_{12}{ }^{\mathrm{a}}$ & $\mathrm{B}_{14}{ }^{\mathrm{a}}$ & $\mathrm{B}_{16}{ }^{\mathrm{a}}$ \\
\hline CHO-K1 & $>1000$ & $108 \pm 5$ & $16 \pm 2$ & $14 \pm 1$ & $7 \pm 1$ & $3.5 \pm 0.1$ & $2.9 \pm 0.2$ & $29 \pm 3$ & $24 \pm 4$ & $15 \pm 1$ \\
\hline
\end{tabular}

\section{Experimental Section}

\subsection{Synthesis}

The pyridinium-4-aldoxime salts 10-14 were prepared by reaction of 4-pyridinealdoxime (1; $8.19 \mathrm{mmol})$ with 1-bromoalkane $(2 ; 12.46 \mathrm{mmol})$ in $\mathrm{CH}_{3} \mathrm{CN}(12 \mathrm{~mL})$, as shown in Scheme 1 . The mixture of 4-pyridinealdoxime with 1-bromoalkane in $\mathrm{CH}_{3} \mathrm{CN}$ was stirred under reflux for $48 \mathrm{~h}$, and the prepared salts were obtained as white crystals by crystallization from acetone, filtered, washed with acetone and allowed to dry at room temperature The second method for the synthesis (Scheme 1) of monoquaternary pyridinium-4-aldoxime salts (3-9) was as follows: Pure 4-pyridinealdoxime (1; 1eq $8.19 \mathrm{mmol})$ in dry ethanol $(30 \mathrm{~mL})$ was mixed with 1-bromoalkane $(2 ; 1,4$ eq $12.46 \mathrm{mmol})$. The mixture was refluxed for $48 \mathrm{~h}$. The solution was evaporated under reduced pressure and the crude oily product was recrystallized from ethyl acetate, filtered, washed with ethyl acetate, and allowed to dry at room temperature.

The progress of the reaction was monitored y TLC (mobile phase ethyl acetate/methanol = 100:1). All prepared products 9-15 were characterized by ${ }^{1} \mathrm{H}-\mathrm{NMR},{ }^{13} \mathrm{C}-\mathrm{NMR}$, elementary analysis and MS analysis.

Acquired yields (\%), melting points (Boetius, uncorrected) and Clog $P$ of prepared salts 9-15 are summarized in Table 1. All chemicals were reagent or higher grade of purity and were purchased from Sigma-Aldrich. The progress of the reaction was checked by Thin Layer Chromatography (TLC) (Merck Milipore Silica gel 60G/UV254, Darmstadt, Germany) with UV detection using wavelength $254 \mathrm{~nm}$. The ${ }^{1} \mathrm{H}-\mathrm{NMR}$ and ${ }^{13} \mathrm{C}-\mathrm{NMR}$ spectra were recorded with a Varian Mercury-VxBB 300 with frequencies $300.07 \mathrm{MHz}$ for ${ }^{1} \mathrm{H}$ and $75.46 \mathrm{MHz}$ for ${ }^{13} \mathrm{C}$. For ${ }^{1} \mathrm{H} \delta$ are given in parts per million (ppm) 
relative to $\operatorname{DMSO}(\delta=2.50)$ and for ${ }^{13} \mathrm{C}$ relative to DMSO $(\delta=39.43) . \log P$ and $C \log P$ were calculated with PC software CS ChemBioDraw Ultra 13.0 (CambridgeSoft, Cambridge, MA, USA).

4-Hydroxyiminomethyl-1-octylpyridinium bromide (9). ${ }^{1} \mathrm{H}-\mathrm{NMR}\left(300 \mathrm{MHz}, \mathrm{DMSO}-d_{6}\right): \delta 12.82$ (s, $1 \mathrm{H}, \mathrm{OH}) ; 9.08(\mathrm{~d}, J=6.3 \mathrm{~Hz}, 2 \mathrm{H}, 2 \times \mathrm{ArH}) ; 8.44(\mathrm{~s}, 1 \mathrm{H}, \mathrm{CH}) ; 8.24(\mathrm{~d}, J=6.7 \mathrm{~Hz}, 2 \mathrm{H}, 2 \times \mathrm{ArH}) ; 4.57$ (t, $\left.J=7.3 \mathrm{~Hz}, 2 \mathrm{H}, \mathrm{NCH}_{2}-\right) ; 1.97-1.81\left(\mathrm{~m}, 2 \mathrm{H}, \mathrm{CH}_{2}\right) ; 1.35-1.16\left(\mathrm{~m}, 10 \mathrm{H}, 5 \times \mathrm{CH}_{2}\right) ; 0.85(\mathrm{t}, J=6.5 \mathrm{~Hz}$, $3 \mathrm{H}, \mathrm{CH}_{3}$ ). ${ }^{13} \mathrm{C}-\mathrm{NMR}\left(75 \mathrm{MHz}, \mathrm{DMSO}-d_{6}\right): \delta 148.5,145.2,145.1,124.2,60.4,49.2,31.5,30.7,28.9$, 25.5, 22.3, 14.1. ESI-MS: $m / z 235.00\left[\mathrm{M}^{+}\right]$(counted for: $\left[\mathrm{C}_{14} \mathrm{H}_{23} \mathrm{~N}_{2} \mathrm{O}\right]^{+}$235,18). Anal. Calcd. for $\mathrm{C}_{14} \mathrm{H}_{23} \mathrm{BrN}_{2} \mathrm{O}: 53.34 \% \mathrm{C} ; 7.35 \% \mathrm{H} ; 8.89 \%$ N. Found: $54.23 \% \mathrm{C} ; 7.31 \% \mathrm{H} ; 8.22 \% \mathrm{~N}$.

1-Decyl-4-hydroxyiminomethylpyridinium bromide (10). ${ }^{1} \mathrm{H}-\mathrm{NMR}\left(300 \mathrm{MHz}, \mathrm{DMSO}-d_{6}\right): \delta 12.80$ (s, $1 \mathrm{H}, \mathrm{OH}) ; 9.08(\mathrm{~d}, J=6.4 \mathrm{~Hz}, 2 \mathrm{H}, 2 \times \mathrm{ArH}) ; 8.43(\mathrm{~s}, 1 \mathrm{H}, \mathrm{CH}) ; 8.23(\mathrm{~d}, J=6.6 \mathrm{~Hz}, 2 \mathrm{H}, 2 \times \mathrm{ArH}) ; 4.57$ $\left(\mathrm{t}, J=7.3 \mathrm{~Hz}, 2 \mathrm{H}, \mathrm{NCH}_{2}\right) ; 1.96-1.81\left(\mathrm{~m}, 2 \mathrm{H}, \mathrm{CH}_{2}\right) ; 1.33-1.17\left(\mathrm{~m}, 14 \mathrm{H}, 7 \times \mathrm{CH}_{2}\right) ; 0.83$ (t, $\left.J=6.6 \mathrm{~Hz}, 3 \mathrm{H}, \mathrm{CH}_{3}\right) .{ }^{13} \mathrm{C}-\mathrm{NMR}\left(75 \mathrm{MHz}, \mathrm{DMSO}-d_{6}\right): \delta 148.5,145.2,145.1,124.2,60.4,49.2,31.5$, 30.7, 29.1, 28.9, 25.5, 22.3, 14.1. ESI-MS: $m / z 263.00\left[\mathrm{M}^{+}\right]$(counted for: $\left[\mathrm{C}_{16} \mathrm{H}_{27} \mathrm{~N}_{2} \mathrm{O}\right]^{+} 263,21$ ). Anal. Calcd. for $\mathrm{C}_{16} \mathrm{H}_{27} \mathrm{BrN}_{2} \mathrm{O}: 55.98 \%$ C; $7.93 \% \mathrm{H} ; 8.16 \%$ N. Found: $55.38 \%$ C; $7.62 \% \mathrm{H} ; 8.15 \%$ N.

1-Dodecyl-4-hydroxyiminomethylpyridinium bromide (11). ${ }^{1} \mathrm{H}-\mathrm{NMR}\left(300 \mathrm{MHz}, \mathrm{DMSO}-d_{6}\right): \delta 12.80$ (s, 1H, OH); 9.09 (d, $J=6.4 \mathrm{~Hz}, 2 \mathrm{H}, 2 \times \mathrm{ArH}) ; 8.43(\mathrm{~s}, 1 \mathrm{H}, \mathrm{CH}) ; 8.23(\mathrm{~d}, J=6.7 \mathrm{~Hz}, 2 \mathrm{H}, 2 \times \mathrm{ArH})$; $4.57\left(\mathrm{t}, J=7.3 \mathrm{~Hz}, 2 \mathrm{H}, \mathrm{NCH}_{2}\right) ; 1.97-1.80\left(\mathrm{~m}, 2 \mathrm{H}, \mathrm{CH}_{2}\right) ; 1.32-1.16\left(\mathrm{~m}, 18 \mathrm{H}, 9 \times \mathrm{CH}_{2}\right) ; 0.83$ (t, $\left.J=6.7 \mathrm{~Hz}, 3 \mathrm{H}, \mathrm{CH}_{3}\right) .{ }^{13} \mathrm{C}-\mathrm{NMR}\left(75 \mathrm{MHz}, \mathrm{DMSO}-d_{6}\right): \delta 148.5,145.2,145.1,124.2,60.4,49.2,31.5$, 30.7, 29.1, 29.0, 28.9, 28.8, 28.6, 25.5, 22.3, 14.2. ESI-MS: $m / z 291.00\left[\mathrm{M}^{+}\right]$(counted for: $\left[\mathrm{C}_{18} \mathrm{H}_{31} \mathrm{~N}_{2} \mathrm{O}\right]^{+}$291.24). Anal. Calcd. for $\mathrm{C}_{18} \mathrm{H}_{31} \mathrm{BrN}_{2} \mathrm{O}: 58.22 \% \mathrm{C} ; 8.41 \% \mathrm{H} ; 7.54 \%$ N. Found: $57.70 \%$ $\mathrm{C} ; 8.44 \% \mathrm{H} ; 7.54 \% \mathrm{~N}$.

4-Hydroxyiminomethyl-1-tetradecylpyridinium bromide (12). ${ }^{1} \mathrm{H}-\mathrm{NMR}(300 \mathrm{MHz}$, DMSO-d6): $\delta 12.81$ $(\mathrm{s}, 1 \mathrm{H}, \mathrm{OH}) ; 9.07(\mathrm{~d}, J=6.4 \mathrm{~Hz}, 2 \mathrm{H}, 2 \times \mathrm{ArH}) ; 8.43(\mathrm{~s}, 1 \mathrm{H}, \mathrm{CH}) ; 8.23(\mathrm{~d}, J=6.5 \mathrm{~Hz}, 2 \mathrm{H}$, $2 \times \mathrm{ArH}) ; 4.56\left(\mathrm{t}, J=7.2 \mathrm{~Hz}, 2 \mathrm{H}, \mathrm{NCH}_{2}\right) ; 1.97-1.81\left(\mathrm{~m}, 2 \mathrm{H}, \mathrm{CH}_{2}\right) ; 1.32-1.17\left(\mathrm{~m}, 22 \mathrm{H}, 11 \times \mathrm{CH}_{2}\right)$; $0.83\left(\mathrm{t}, J=6.6 \mathrm{~Hz}, 3 \mathrm{H}, \mathrm{CH}_{3}\right) .{ }^{13} \mathrm{C}-\mathrm{NMR}\left(75 \mathrm{MHz}, \mathrm{DMSO}-d_{6}\right): \delta 148.5,145.3,145.2,124.2,60.2,49.9$, $31.4 ; 30.7,30.1,30.0,29.8,29.7,29.3,29.1,28.6,25.5,22.3,14.1$. ESI-MS: $m / z 319.00\left[\mathrm{M}^{+}\right]$(counted for: $\left[\mathrm{C}_{20} \mathrm{H}_{35} \mathrm{~N}_{2} \mathrm{O}\right]^{+}$319.27). Anal. Calcd. for $\mathrm{C}_{20} \mathrm{H}_{35} \mathrm{BrN}_{2} \mathrm{O}: 60.14 \% \mathrm{C} ; 8.33 \% \mathrm{H} ; 7.01 \% \mathrm{~N}$. Found: $59.65 \% \mathrm{C} ; 8.98 \% \mathrm{H} ; 7.02 \% \mathrm{~N}$.

1-Hexadecyl-4-hydroxyiminomethylpyridinium bromide (13). ${ }^{1} \mathrm{H}-\mathrm{NMR}\left(300 \mathrm{MHz}, \mathrm{DMSO}-d_{6}\right): \delta 12.82$ $(\mathrm{s}, 1 \mathrm{H}, \mathrm{OH}) ; 9.07(\mathrm{~d}, J=6.4 \mathrm{~Hz}, 2 \mathrm{H}, 2 \times \mathrm{ArH}) ; 8.44(\mathrm{~s}, 1 \mathrm{H}, \mathrm{CH}) ; 8.24(\mathrm{~d}, J=6.7 \mathrm{~Hz}, 2 \mathrm{H}$, $2 \times \mathrm{ArH}) ; 4.57\left(\mathrm{t}, J=7.2 \mathrm{~Hz}, 2 \mathrm{H}, \mathrm{NCH}_{2}\right) ; 1.96-1.82\left(\mathrm{~m}, 2 \mathrm{H}, \mathrm{CH}_{2}\right) ; 1.32-1.18\left(\mathrm{~m} .26 \mathrm{H}, 13 \times \mathrm{CH}_{2}\right)$; $0.84\left(\mathrm{t}, J=6.4 \mathrm{~Hz}, 3 \mathrm{H}, \mathrm{CH}_{3}\right) .{ }^{13} \mathrm{C}-\mathrm{NMR}\left(75 \mathrm{MHz}, \mathrm{DMSO}-d_{6}\right): \delta 148.5,145.3,145.2,124.2,60.2,49.9$, $31.4 ; 30.7,30.1,30.0,29.8,29.7,29.6,29.3,29.1,28.9,28.6,25.5,22.3,14.1$. ESI-MS: $m / z 347.00$ $\left[\mathrm{M}^{+}\right]$(counted for: $\left[\mathrm{C}_{22} \mathrm{H}_{39} \mathrm{~N}_{2} \mathrm{O}\right]^{+} 347.31$ ). Anal. Calcd. for $\mathrm{C}_{22} \mathrm{H}_{39} \mathrm{BrN}_{2} \mathrm{O}: 61.81 \% \mathrm{C} ; 9.20 \% \mathrm{H} ; 6.55 \% \mathrm{~N}$. Found: $61.52 \% \mathrm{C} ; 9.24 \% \mathrm{H} ; 6.64 \% \mathrm{~N}$.

4-Hydroxyiminomethyl-1-octadecylpyridinium bromide (14). ${ }^{1} \mathrm{H}-\mathrm{NMR}$ (300 MHz, DMSO-d6): $\delta 12.82$ (s, $1 \mathrm{H}, \mathrm{OH}) ; 9.08$ (d, $J=6.4 \mathrm{~Hz}, 2 \mathrm{H}, 2 \times \mathrm{ArH}) ; 8.44(\mathrm{~s}, 1 \mathrm{H}, \mathrm{CH}) ; 8.24(\mathrm{~d}, J=6.6 \mathrm{~Hz}, 2 \mathrm{H}, 2 \times \mathrm{ArH})$; 
$4.57\left(\mathrm{t}, J=7.3 \mathrm{~Hz}, 2 \mathrm{H}, \mathrm{NCH}_{2}\right) ; 1.97-1.81\left(\mathrm{~m}, 2 \mathrm{H}, \mathrm{CH}_{2}\right) ; 1.35-1.15\left(\mathrm{~m}, 30 \mathrm{H}, 15 \times \mathrm{CH}_{2}\right) ; 0.84$ (t, $\left.J=6.7 \mathrm{~Hz}, 3 \mathrm{H}, \mathrm{CH}_{3}\right) .{ }^{13} \mathrm{C}-\mathrm{NMR}\left(75 \mathrm{MHz}, \mathrm{DMSO}-d_{6}\right): \delta 148.5,145.3,145.2,124.2,60.2,49.9,31.4$; $30.7,30.1,30.0,29.8,29.7,29.6,29.5,29.3,29.2,29.1,28.9,28.6,25.5,22.3,14.1$. ESI-MS: $\mathrm{m} / \mathrm{z}$ $375.34\left[\mathrm{M}^{+}\right]$(counted for: $\left[\mathrm{C}_{24} \mathrm{H}_{43} \mathrm{~N}_{2} \mathrm{O}\right]^{+} 375.34$ ). Anal. Calcd. for $\mathrm{C}_{24} \mathrm{H}_{43} \mathrm{BrN}_{2} \mathrm{O}: 63.28 \% \mathrm{C} ; 9.51 \% \mathrm{H}$; $6.15 \%$ N. Found: $62.66 \%$ C; $9.67 \% \mathrm{H} ; 6.09 \%$ N.

Eicosyl-4-hydroxyiminomethylpyridinium bromide (15). ${ }^{1} \mathrm{H}-\mathrm{NMR}\left(300 \mathrm{MHz}, \mathrm{DMSO}-d_{6}\right): \delta 12.82$ (s, $1 \mathrm{H}, \mathrm{OH}) ; 9.05(\mathrm{~d}, J=6.2 \mathrm{~Hz}, 2 \mathrm{H}, 2 \times \mathrm{ArH}) ; 8.43(\mathrm{~s}, 1 \mathrm{H}, \mathrm{CH}) ; 8.23(\mathrm{~d}, J=6.5 \mathrm{~Hz}, 2 \mathrm{H}, 2 \times \mathrm{ArH}) ; 4.56$ (t, $\left.J=7.3 \mathrm{~Hz}, 2 \mathrm{H}, \mathrm{NCH}_{2}\right) ; 1.98-1.80\left(\mathrm{~m}, 2 \mathrm{H}, \mathrm{CH}_{2}\right) ; 1.33-1.17\left(\mathrm{~m}, 34 \mathrm{H}, 17 \times \mathrm{CH}_{2}\right) ; 0.85(\mathrm{t}, J=6.6 \mathrm{~Hz}, 3 \mathrm{H}$, $\left.\mathrm{CH}_{3}\right) .{ }^{13} \mathrm{C}-\mathrm{NMR}(75 \mathrm{MHz}$, DMSO-d6): $\delta$ 148.5, 145.3, 145.2, 124.2, 60.2, 49.9, 31.4; 30.7, 30.1, 30.0, 29.8, 29.7, 29.6, 29.5, 29.4, 29.3, 29.2, 29.1, 28.9, 28.8, 28.6, 25.5, 22.3, 14.1. ESI-MS: $m / z 403.50$ $\left[\mathrm{M}^{+}\right]$(counted for: $\left[\mathrm{C}_{26} \mathrm{H}_{47} \mathrm{~N}_{2} \mathrm{O}\right]^{+}$403.37). Anal. Calcd. for $\mathrm{C}_{26} \mathrm{H}_{47} \mathrm{BrN}_{2} \mathrm{O}$ : $64.58 \% \mathrm{C} ; 9.80 \% \mathrm{H} ; 5.79 \%$ N. Found: $63.94 \% \mathrm{C} ; 9.80 \% \mathrm{H} ; 5.76 \% \mathrm{~N}$.

\subsection{HPLC Analysis}

After the preparation of the whole set of 4-PA salts differing in the length of alkyl chain, we have developed an appropriate method for their resolution in a mixture using HPLC. The HPLC system consisted of a P200 gradient pump (Spectra-Physics Analytical, Fremont, CA, USA), a 7125 injection valve-10 $\mu \mathrm{L}$ loop (Rheodyne, Cotati, CA, USA), a UV1000 detector (Spectra-Physics Analytical, Fremont, CA, USA) and CSW Chromatography Station 1.5 software (DataApex, Praha, Czech republic). For analyses a $250 \times 4.6 \mathrm{~mm}$ I.D. Waters Spherisorb Cyano $(5 \mu \mathrm{m})$ column was used (Supelco Inc., Bellefonte, PA, USA). The mobile phase was $45 \%$ acetonitrile and $55 \%$ water. This mixture was prepared as a $0.1 \mathrm{M}$ sodium acetate solution. Finally, the $\mathrm{pH}$ was adjusted with acetic acid to 5000. It was delivered isocratically at a flow-rate of $1 \mathrm{~mL} / \mathrm{min}$. The absorbance was measured at $257 \mathrm{~nm}$. Retention times are summarized in Table 1.

\subsection{In-Vitro Antimicrobial Testing}

\subsubsection{Antifungal Activity}

In vitro antifungal activity of the prepared compounds was evaluated on a panel of eight clinical isolates of fungi, four yeasts (C. albicans ATCC 44859, C. krusei E28, C. tropicalis 156, C. glabrata 20/I) and four filamentous fungi (Trichosporon asahii 1188, Aspergillus fumigatus 231, Absidia corymbifera 272, Trichophyton mentagrophytes 445). All strains were part of the collection of fungal strains and are deposited at the Department of Biological and Medical Sciences, Faculty of Pharmacy, Charles University, Hradec Kralove, Czech Republic. The ATCC strains C. albicans ATCC 90028, C. parapsilosis ATCC 22019, and C. krusei ATCC 6258 served as the quality control strains.

All the isolates were maintained on Sabouraud dextrose agar prior to being tested. Minimum inhibitory concentration (MIC) was determined by the modified microdilution format of the CLSI M27-A3 and M38-A2 for yeasts and filamentous fungi, respectively [42,43]. Dimethyl sulfoxide (Sigma, Prague, Czech Republic) served as a diluent for all compounds and its final concentration did not exceed 2\%. RPMI 1640 (KlinLab, Prague, Czech Republic) medium supplemented with L-glutamine 
and buffered with $0.165 \mathrm{M}$ morpholinepropanesulfonic acid (Sigma-Aldrich, Prague) to $\mathrm{pH} 7.0$ by $10 \mathrm{M}$ $\mathrm{NaOH}$ was used as a test medium. The wells of the microdilution tray contained $200 \mu \mathrm{L}$ of the RPMI 1640 medium with two fold serial dilutions of the prepared compounds $(500-0.49 \mu \mathrm{mol} / \mathrm{L})$ and were inoculated with $10 \mu \mathrm{L}$ of suspension. The fungal inoculum in RPMI 1640 was prepared to give a final concentration of $5 \times 10^{3} \pm 0.2 \mathrm{cfu} / \mathrm{mL}$ and $5 \times 10^{4} \pm 0.5 \mathrm{cfu} / \mathrm{mL}$ for yeasts and moulds, respectively. The trays were incubated at $36{ }^{\circ} \mathrm{C} \pm 1{ }^{\circ} \mathrm{C}$ and MIC was read visually and spectrophotometrically (OD $450 \mathrm{~nm}$ ) for filamentous fungi and yeasts respectively after 24 and $48 \mathrm{~h}$. The MIC values for the dermatophytic strain (T. mentagrophytes) were determined after $72 \mathrm{~h}$ and $120 \mathrm{~h}$. The MICs were defined as $80 \%$ inhibition ( $\mathrm{IC}_{80}$ ) of the growth of control. Minimum fungicidal concentration (MFC) was established for all compounds tested as the concentration which provided a decrease in the number of colonies by $\geq 99.9 \%$ after subculturing of a $100 \mu \mathrm{L}$ aliquot of each well with maximum growth of $20 \%$ of control.

\subsubsection{Antibacterial Activity}

The in vitro antibacterial activity of the prepared compounds was tested on a panel of eight bacterial strains (Staphylococcus aureus CCM 4516/08, S. aureus MRSA H 5996/08, S. epidermidis HK6966/08, Enterococcus sp. HK14365/08, Escherichia coli CCM 4517, Klebsiella pneumoniae D 11750/08, K. pneumoniae J 14368/08, and Pseudomonas aeruginosa CCM 1961). The ATCC strains also served as the quality control strains; the rest of them were clinical isolates from the patients and are deposited at the Department of Biological and Medical Sciences, Faculty of Pharmacy, Charles University, Hradec Kralove, Czech Republic. Before testing the strains were passaged on MuellerHinton Agar (HiMedia, Cadersky-Envitek, Prague, Czech Republic).

Minimum inhibitory concentration (MIC) of the prepared compounds was determined by the microdilution broth method modified according to standard M07-A07 [44]. Mueller-Hinton Broth (MH, HiMedia, Cadersky-Envitek, Prague, Czech Republic) adjusted to $\mathrm{pH} 7.4( \pm 0.2)$ was used as the test medium. DMSO served as a diluent for all compounds and its final concentration did not exceed $2 \%$ in the test medium. The wells of the microdilution tray contained $200 \mu \mathrm{L}$ of the MH broth with twofold serial dilutions of the compounds $(500-0.49 \mu \mathrm{mol} / \mathrm{L})$ and were inoculated with $10 \mu \mathrm{L}$ of bacterial suspension. A bacterial inoculum in sterile water was prepared to match $0.5 \mathrm{McFarland}$ scale $\left(1.5 \times 10^{8} \mathrm{CFU} / \mathrm{mL}\right)$. The MIC values were read visually after $24 \mathrm{~h}$ and $48 \mathrm{~h}$ incubation at $36{ }^{\circ} \mathrm{C} \pm 1{ }^{\circ} \mathrm{C}$; for Pseudomonas aeruginosa CCM 1961, the MIC was determined after $72 \mathrm{~h}$ and $120 \mathrm{~h}$ of incubation. The MIC was defined as complete inhibition of growth. Minimum bactericidal concentration (MBC) was established for all compounds tested as the concentration that provided a decrease in the number of colonies by $\geq 99.9 \%$ after subculturing of a $100 \mu \mathrm{L}$ aliquot of each well without visible growth.

\subsection{Cytotoxicity}

Standard MTT assay (Sigma Aldrich, Prague, Czech Republic) was used according to the manufacturer's protocol on the CHO-K1 cell-line (Chinese hamster ovary, ECACC, Salisbury, UK) in order to compare the cytotoxic effect of the studied compounds. The cells were cultured according to ECACC recommended conditions and seeded at a density of 8000 per well. Briefly, the tested compounds were dissolved in DMSO and subsequently in the growth medium (F-12) supplemented 
with $10 \%$ FBS and $1 \%$ penicillin/streptomycin so that the final concentration of DMSO did not exceed $0.5 \%(\mathrm{v} / \mathrm{v})$. Cells were exposed to the tested compounds for $24 \mathrm{~h}$. The medium was then replaced by a medium containing $10 \mu \mathrm{M}$ of MTT and the cells were allowed to produce formazan for another $3 \mathrm{~h}$ under surveillance. Thereafter, the medium with MTT was removed and crystals of formazan were dissolved in DMSO $(100 \mu \mathrm{L})$. Cell viability was assessed spectrophotometrically by the amount of formazan produced. Absorbance was measured at $570 \mathrm{~nm}$ with $650 \mathrm{~nm}$ reference wavelength on Synergy HT (BioTek, Winooski, VT, USA). IC 50 was then calculated from the control-subtracted triplicates using non-linear regression (four parameters) of GraphPad Prism 5 software. The final IC50 and SEM value was obtained as the mean of three independent measurements.

\section{Conclusions}

The whole set of pyridine-4-aldoxime based quaternary ammonium salts with differing length of alkyl side chain was synthesized (9-15). We have described two possible conditions for the preparation. Both methods are usable for the preparation and no significant difference in the yields was observed. The compounds were analyzed by NMR, EA and MS analysis and the $\log P$ was calculated for each compound. Furthermore, an HPLC experimental protocol was found that was fully applicable for purity evaluation and could be easily used for characterization of mixtures of all homologues. Antimicrobial (antifungal and antibacterial) activity evaluation confirmed that compounds having an alkyl chain of $\mathrm{C}_{12}-\mathrm{C}_{16}$ outperformed the rest of the tested compounds. Yeast-type fungi were the most sensitive towards those analogues whereas the $\mathrm{C}_{16}$ analogue was completely ineffective against filamentous fungi. Antibacterial assessment revealed versatility of $\mathrm{C}_{14}$ and relatively high efficacy of $\mathrm{C}_{16}$ against $\mathrm{G}+$ strains and $\mathrm{C}_{14}$ against $\mathrm{G}^{-}$strains. Notably, none of the studied compounds exceeded the efficacy and versatility of the benzalkonium $\mathrm{C}_{12}$ analogue, and benzalkonium analogues also showed a lower effect in the cell viability assay.

\section{Acknowledgments}

The publication is co-financed by the European Social Fund and the state budget of the Czech Republic. Post-doctoral Project no. CZ.1.07/2.3.00/30.0061, CZ.1.07/2.3.00/30.0016. This work was also supported by long development plan 1011 and MH CZ_-DRO (UHHK, 00179906). We wish to thank Jiri Kunes (Faculty of Pharmacy, Charles University in Prague, Hradec Kralove) for measurement of NMR spectra.

\section{Author Contributions}

J.M. and D.M. were responsible for the preparation of the compounds, identification and purity analysis. J.M. also ensured biological testing on microbial strains and manuscript preparation. R.D. performed HPLC analysis. O.S. and M.P. were responsible for the cell viability testing and structure-activity relationship evaluation. M.D. and K.K. participated on the design of new compounds, management of the study, were responsible for the funding, final correction of the manuscript and correspondence. 


\section{Conflicts of Interest}

The authors declare no conflict of interest. The founding sponsors had no role in the design of the study; in the collection, analyses, or interpretation of data; in the writing of the manuscript, and in the decision to publish the results.

\section{References}

1. Singh, S.; Bhadani, A.; Kataria, H.; Kaur, G.; Kamboj, R. Synthesis of glycerol-based pyridinium surfactants and appraisal of their properties. Ind. Eng. Chem. Res. 2009, 48, 1673-1677.

2. Earle, M.J.; Katdare, S.P.; Seddon, K.R. Paradigm confirmed: The first use of ionic liquids to dramatically influence the outcome of chemical reactions. Org. Lett. 2004, 6, 707-710.

3. Price, P.B. Benzalkonium chloride (zephiran chloride) as a skin disinfectant. Arch. Surg. 1950, 61, 23-33.

4. Semmler, A.; Kohler, H.H. Surface properties of alkylpyridinium chlorides and the applicability of the pendant drop technique. J. Colloid Interface Sci. 1999, 218, 137-144.

5. Akbas, H.; Kartal, C.C.I. Reactive orange 16-dodecylpyridinium chloride interactions in electrolytic solutions. Spectrochim. Acta A Mol. Biomol. Spectrosc. 2006, 65, 95-99.

6. Rodriguez-Morales, S.; Compadre, R.L.; Castillo, R.; Breen, P.J.; Compadre, C.M. 3D-QSAR, synthesis, and antimicrobial activity of 1-alkylpyridinium compounds as potential agents to improve food safety. Eur. J. Med. Chem. 2005, 40, 840-849.

7. Sivak, A.; Goyer, M.; Perwak, J.; Thayer, P. Environmental and Human Health Aspects of Commercially Important Surfactants. In Solution Behaviour of Surfactants (Theoretical and Applied Aspects); Mittal, K.L., Fendler, E.J., Eds.; Plenum Press: New York, NY, USA, 1982; Volume 1, pp. 161-188.

8. Kuca, K.; Bielavska, M.; Cabal, J.; Dohnal, V. Determination of benzalkonium bromide homologues in disinfection products using high-performance liquid chromatography. Anal. Lett. 2005, 38, 673-682.

9. Kuca, K.; Marek, J.; Stodulka, P.; Musilek, K.; Hanusova, P.; Hrabinova, M.; Jun, D. Preparation of benzalkonium salts differing in the length of a side alkyl chain. Molecules 2007, 12, 2341-2347.

10. Wong, Y.L.; Hubieki, M.P.; Curfman, C.L.; Doncel, G.F.; Dudding, T.C.; Savle, P.S.; Gandour, R.D. A structure-activity study of spermicidal and anti-hiv properties of hydroxylated cationic surfactants. Bioorg. Med. Chem. 2002, 10, 3599-3608.

11. Lin, J.; Qiu, S.Y.; Lewis, K.; Klibanov, A.M. Bactericidal properties of flat surfaces and nanoparticles derivatized with alkylated polyethylenimines. Biotechnol. Prog. 2002, 18, 1082-1086.

12. Tiller, J.C.; Liao, C.J.; Lewis, K.; Klibanov, A.M. Designing surfaces that kill bacteria on contact. Proc. Natl. Acad. Sci. USA 2001, 98, 5981-5985.

13. Faraj, J.A.; Dorati, R.; Schoubben, A.; Worthen, D.; Selmin, F.; Capan, Y.; Leung, K.; DeLuca, P.P. Development of a peptide-containing chewing gum as a sustained release antiplaque antimicrobial delivery system. AAPS PharmSciTech 2007, 8, E177-E185.

14. Tanzer, J.M.; Slee, A.M.; Kamay, B.; Scheer, E.R. In vitro evaluation of seven cationic detergents as antiplaque agents. Antimicrob. Agents Chemother. 1979, 15, 408-414. 
15. Shelton, R.S.; Vancampen, M.G.; Tilford, C.H.; Lang, H.C.; Nisonger, L.; Bandelin, F.J.; Rubenkoenig, H.L. Quaternary ammonium salts as germicides 1. Non-acylated quaternary ammonium salts derived from aliphatic amines. J. Am. Chem. Soc. 1946, 68, 753-755.

16. Li, Y.B.; Slavik, M.F.; Walker, J.T.; Xiong, H. Pre-chill spray of chicken carcasses to reduce salmonella typhimurium. J. Food Sci. 1997, 62, 605-607.

17. Brill, F.; Goroncy-Bermes, P.; Sand, W. Influence of growth media on the sensitivity of staphylococcus aureus and pseudomonas aeruginosa to cationic biocides. Int. J. Hyg. Environ. Health 2006, 209, 89-95.

18. Dwars, T.; Paetzold, E.; Oehme, G. Reactions in micellar systems. Angewa. Chem. Int. Ed. 2005, 44, 7174-7199.

19. Ghosh, K.K.; Kolay, S.; Bal, S.; Satnami, M.L.; Quagliotto, P.; Dafonte, P.R. Effect of cationic gemini surfactants on the hydrolysis of carboxylate and phosphate esters using hydroxamate ions. Coll. Polym. Sci. 2008, 286, 293-303.

20. Ghosh, K.K.; Satnami, M.L.; Sinha, D. Dephosphorylation of paraoxon by hydroxamate ions in micellar media. Tetrahedron. Lett. 2004, 45, 9103-9105.

21. Singh, N.; Ghosh, K.K.; Marek, J.; Kuca, K. Hydrolysis of carboxylate and phosphate esters using monopyridinium oximes in cationic micellar media. Int. J. Chem. Kinet. 2011, 43, 569-578.

22. Cabal, J.; Kuca, K.; Sevelova-Bartosova, L.; Dohnal, V. Cyclodextrines as functional agents for decontamination of the skin contaminated by nerve agents. Acta Medica (Hradec Kral.) 2004, 47, $115-118$.

23. Tiwari, S.; Ghosh, K.K.; Marek, J.; Kuca, K. Comparative study of nucleophilic efficacy of pralidoxime towards phosphorus, sulfur and thiophosphorus based esters. React. Kinet. Catal. Lett. 2009, 98, 91-97.

24. Tiwari, S.; Ghosh, K.K.; Marek, J.; Kuca, K. Functionalized surfactant mediated reactions of carboxylate, phosphate and sulphonate esters. J. Phys. Org. Chem. 2010, 23, 519-525.

25. Tiwari, S.; Ghosh, K.K.; Marek, J.; Kuca, K. Cationic micellar-catalyzed hydrolysis of pesticide fenitrothion using alpha-nucleophiles. Lett. Drug Des. Discov. 2010, 7, 194-199.

26. Tiwari, S.; Kolay, S.; Ghosh, K.K.; Kuca, K.; Marek, J. Kinetic study of the reactions of p-nitrophenyl acetate and $p$-nitrophenyl benzoate with oximate nucleophiles. Int. J. Chem. Kinet. 2009, 41, 57-64.

27. Fisicaro, E.; Pelizzetti, E.; Viscardi, G.; Quagliotto, P.L.; Trossarelli, L. Thermodynamic properties of aqueous micellar solutions of $\mathrm{N}-(1 \mathrm{H}, 1 \mathrm{H}, 2 \mathrm{H}, 2 \mathrm{H}$ perfluorooctyl)pyridinium chloride and $\mathrm{N}-(1 \mathrm{H}, 1 \mathrm{H}, 2 \mathrm{H}, 2 \mathrm{H}$ perfluorodecyl)pyridinium chloride. In Colloids and Surfaces A: Physicochemical and Engineering Aspects, Proceedings of the ACS Symposium on Colloid and Surface Chemistry of Fluorocarbons and Highly Fluorinated Amphiphiles at 205th Annual ACS Meeting, Denver, CO, USA, 28 March-2 April 1993, Franses, E.I., Weers, J.G., Eds.; Elsevier: London, UK, 1994; Volume 84, pp. 59-70.

28. Fisicaro, E.; Ghiozzi, A.; Pelizzetti, E.; Viscardi, G.; Quagliotto, P.L. Effect of the counterion on thermodynamic properties of aqueous micellar solutions of 1-(3,3,4,4,5,5,6,6,6-nonafluorohexyl) pyridinium halides: Ii. Apparent and partial molar enthalpies and osmotic coefficients at $313 \mathrm{k}$. J. Colloid Interface Sci. 1996, 184, 147-154. 
29. Fisicaro, E.; Biemmi, M.; Compari, C.; Duce, E.; Peroni, M. Thermodynamics of aqueous solutions of dodecyldimethylethylammonium bromide. J. Colloid Interface Sci. 2007, 305, 301-307.

30. Kuca, K.; Kivala, M.; Dohnal, V. A general method for the quaternization of $N, N$-dimethyl benzylamines with long chain N-alkylbromides. J. Appl. Biomed. 2004, 2, 195-198.

31. Spilovska, K.; Korabecny, J.; Kral, J.; Horova, A.; Musilek, K.; Soukup, O.; Drtinova, L.; Gazova, Z.; Siposova, K.; Kuca, K. 7-Methoxytacrine-adamantylamine heterodimers as cholinesterase inhibitors in Alzheimer's disease treatment-synthesis, biological evaluation and molecular modeling studies. Molecules 2013, 18, 2397-2418.

32. Korabecny, J.; Soukup, O.; Dolezal, R.; Spilovska, K.; Nepovimova, E.; Andrs, M.; Nquyen, T.D.; Jun, D.; Musilek, K.; Kucerova-Chlupacova, M.; Kuca, K. From pyridinium-based to central active acetylcholinesterase reactivators. Mini Rev. Med. Chem. 2014, 14, 215-221.

33. Malinak, D.; Dolezal, R.; Marek, J.; Salajkova, S.; Soukup, O.; Vejsova, M.; Korabecny, J.; Honegr, J.; Penhaker, M.; Musilek, K.; et al. 6-Hydroxyquinolinium salts differing in the length of alkyl side-chain: Synthesis and antimicrobial activity. Bioorg. Med. Chem. Lett. 2014, 24, 5238-5241.

34. Jordan, D.; Tan, E.; Hegh, D. Synthesis, characterization and conductivity of quaternary nitrogen surfactants modified by the addition of a hydroxymethyl substructure on the head group. J. Surfactants Deterg. 2012, 15, 587-592.

35. Kamboj, R.; Singh, S.; Chauhan, V. Synthesis, characterization and surface properties of $N$-(2-hydroxyalkyl)-N'-(2-hydroxyethyl)imidazolium surfactants. Coll. Surf. A Physicochem. Eng. Asp. 2014, 441, 233-241.

36. Li, Y.L.; Li, Q.X.; Zhi, L.F.; Zhang, M.H. Synthesis, characterization and surface activity of trioctyl hydroxyethyl ammonium chloride. Tenside Surfactants Deterg. 2011, 48, 305-307.

37. Marek, J.; Stodulka, P.; Cabal, J.; Soukup, O.; Pohanka, M.; Korabecny, J.; Musilek, K.; Kuca, K. Preparation of the pyridinium salts differing in the length of the $\mathrm{N}$-alkyl substituent. Molecules 2010, 15, 1967-1972.

38. Marek, J.; Buchta, V.; Soukup, O.; Stodulka, P.; Cabal, J.; Ghosh, K.K.; Musilek, K.; Kuca, K. Preparation of quinolinium salts differing in the length of the alkyl side chain. Molecules 2012, 17, 6386-6394.

39. Coughlin, R.T.; Tonsager, S.; McGroarty, E.J. Quantitation of metal-cations bound to membranes and extracted lipopolysaccharide of escherichia-coli. Biochemistry 1983, 22, 2002-2007.

40. McDonnell, G.; Russell, A.D. Antiseptics and disinfectants: Activity, action, and resistance. Clin. Microbiol. Rev. 2001, 14, 227-228.

41. Zhong, X.; Guo, J.W.; Fu, S.Q.; Zhu, D.Y.; Peng, J.P. Synthesis, surface property and antimicrobial activity of cationic gemini surfactants containing adamantane and amide groups. J. Surfactants Deterg. 2014, 17, 943-950.

42. Clinical and Laboratory Standards Institute (CLSI). Reference Method for Broth Dilution Antifungal Susceptibility Testing of Yeasts, In Approved Standard-Third Edition; CLSI document M27-A3; CLSI: Wayne, PA, USA, 2008.

43. Clinical and Laboratory Standards Institute (CLSI). Reference Method for Broth Dilution Antifungal Susceptibility Testing of Filamentous Fungi, In Approved Standard-Second Edition; CLSI document M38-A2; CLSI: Wayne, PA, USA, 2008. 
44. Clinical and Laboratory Standards Institute (CLSI). Methods for Dilution Antimicrobial Susceptibility Tests for Bacteria that Grow Aerobically, In Approved Standard-Seventh Edition; CLSI document M7-A7; CLSI: Wayne, PA, USA, 2006.

Sample Availability: Samples of the compounds are available from the authors.

(C) 2015 by the authors; licensee MDPI, Basel, Switzerland. This article is an open access article distributed under the terms and conditions of the Creative Commons Attribution license (http://creativecommons.org/licenses/by/4.0/). 